\title{
ANTI-STRESS ACTION OF SEVERAL ORALLY-GIVEN $\beta$-GLUCANS
}

\author{
Vaclav Vetvicka ${ }^{\text {a*}}$, Zuzana Vancikova ${ }^{\mathrm{b}}$ \\ a University of Louisville, Department of Pathology, Louisville, KY \\ ${ }^{b}$ Paediatric Clinic IPVZ, University Hospital Krc, Prague, Czech Republic \\ E-mail:Vaclav.vetvicka@louisville.edu
}

Received: February 15, 2010; Accepted: August 31, 2010

Keywords: Glucan/Stress/Immunomodulator/Cytokines

Background. $\beta$-Glucans are well-established immunomodulators. Recently, glucans have been found to influence stress-related immunosuppression.

Aim. The aim of this study was to compare the effects of four different types of $\beta$-glucans on immune reactions suppressed by cold- or restrain-induced stress.

Methods. Mice were subjected to restraint and cold stress for various time intervals. The ability of individual glucans to overcome stress-related changes was evaluated after 14 days of feeding.

Results. First, we showed that cold stress caused 38\% decrease in phagocytic activity. While all glucans showed some ability to inhibit stress-related inhibition, only glucan \#300 was able to return the phagocytosis to a normal level. In the control group, feeding with glucans did not alter the level of corticosterone. On the other hand, both types of stress resulted in a significant increase in corticosterone which was blocked to some extent by feeding with glucan. Both types of stress reduced IL-6 secretion but only glucan \#300 managed to keep IL-6 secretion above control levels. The same results were obtained in the case of IL-12.

Conclusion. From our data, we can conclude that, even when all orally-administered glucans helped to restore the stress-related decrease in immune reaction, the level of activity varied widely among individual glucans. In addition, the results suggest that glucans might work via inhibition of corticosterone levels and/or stimulation of cytokine production.

\section{INTRODUCTION}

$\beta 1,3$ Glucans are structurally complex homopolymers of glucose, usually isolated from yeast, fungi and wheat. $\beta 1,3$-glucan's role as a biologically active immunomodulator has been well-documented for more than 50 years. Interest in the immunomodulatory properties of polysaccharides was initially raised after experiments showing that a crude yeast cell preparation stimulated macrophages via activation of the complement system ${ }^{1}$. Further work identified the immunomodulatory active component as $\beta 1,3$-glucan ${ }^{2}$. $\beta$-Glucans show notable physiological effects; this is their most important quality and the reason why so much attention has been focused on them. Numerous studies (currently more than 5,000 publications) have subsequently shown that $\beta 1,3$-glucans, either particulate or soluble, exhibits immunostimulating properties, including antibacterial and anti-tumor activities (for review see ${ }^{3,4}$ ).

In addition to different natural sources, diverse data on the comparison of structure, molecular size, and biological effect can be found in the literature. For example, antitumor activity of schizophyllan is supposedly conditioned by a triple helix presence and a molecular weight higher than $100 \mathrm{kDa}\left(\right.$ ref. $\left.^{5}\right)$. However, the triple helix structure most likely should not be the sole effective form of $\beta$-glucan because alkalic treatment, used in most of isolation procedures, destroys this structure ${ }^{6,7}$. In addition, recent opinions do not confirm established ideas of the necessity of high molecular mass and branching of biologically active $\beta$-glucans. Thirty years ago, Kabat ${ }^{8}$ found that, for antigen polysaccharidic determinants, the size of the binding site on an antibody corresponds to six or seven monosaccharide units. The size of the binding site for $\beta$-glucan - in this case on a receptor of an immunocompetent cell, e.g., the macrophage - also appears to correspond with this number of glucose residues ${ }^{8}$. This considerable heterogeneity of all natural $\beta$-glucans, continues to be the cause of a series of mutually contradicting conclusions. Recently, an attempt was made to solve this problem using semisynthetic and synthetic probes suitable for accurate immunological research ${ }^{9}$. In addition, very few studies comparing various commercially available glucans exist $\mathrm{t}^{10,11}$.

As the glucans are more and more frequently used as an anti-stress agent, we decided to compare four commercially important glucans and their effect on stress.

\section{MATERIAL AND METHODS}

\section{Animals}

Female, 8 week old BALB/c mice were purchased from the Jackson Laboratory (Bar Harbor, ME). All animal work was done according to the University of Louisville IACUC protocol. Animals were sacrificed by $\mathrm{CO}_{2}$ asphyxiation. 


\section{$\beta-1,3$ glucans}

Yeast-derived insoluble glucan \#300 with purity over 96\% was purchased from Transfer Point (Columbia, SC), Now BETA glucan from Now Foods (Bloomingdale, IL) is a mixture of insoluble yeast and soluble mushroom glucans, grain-derived partly soluble Glucagel $\mathrm{T}$ from GraceLinc (Christchurch, New Zealand), the typical carbohydrate content is $85-90 \%$. Epicor is soluble yeastderived glucan from Embria Health Sciences, Ankeny, IA.

\section{Phagocytosis}

The technique employing phagocytosis of synthetic polymeric microspheres was described earlier ${ }^{12,13}$. Briefly: peripheral blood cells were incubated with $0.05 \mathrm{ml}$ of 2-hydroxyethyl methacrylate particles (HEMA; 5x10 $/ \mathrm{ml}$ ). The test tubes were incubated at $37^{\circ} \mathrm{C}$ for $60 \mathrm{~min}$, with intermittent shaking. Smears were stained with Wright stain. The cells with three or more HEMA particles were considered positive. The same smears were also used for evaluation of cell types.

\section{Restrain stress protocol}

Mice were subjected to restraint stress according to a method of Kimura et al. ${ }^{14}$. Briefly, mice were restrained for $6 \mathrm{hr}$ for 5 days (starting at day 9) in a $50 \mathrm{ml}$ conical polypropylene centrifuge tubes in which holes had been drilled. Since the restrained mice could not access food and water, the control group of mice was similarly deprived of food and water. Individual glucans were adminstered orally for 14 days, starting at day 0 .

\section{Cold stress protocol}

Mice were subjected to cold stress ${ }^{15}$ by incubation for $60 \mathrm{~min}$ at $4^{\circ} \mathrm{C}$ for two weeks. For the same time interval, mice received individual glucans administered orally.

\section{Measurement of serum corticosterone}

On day 14, blood samples were collected by venipucture from mice under pentobarbital anesthesia. Obtained sera were collected and stored at $-80^{\circ} \mathrm{C}$ before assay. Serum corticosterone was measured using an ELISA kit (Dignostics Systems Lab, TX) according to the manufacturer's instroctions.

\section{Cytokine production}

Purified spleen cells $\left(2 \times 10^{6} / \mathrm{ml}\right.$ in RPMI 1640 medium with $5 \%$ FCS) were added into wells of a 24-well tissue culture plate. After addition of $1 \mu \mathrm{g}$ of Concanavalin A, cells were incubated for $48 \mathrm{hrs}$. in a humidified incubator $\left(37^{\circ}\right.$ $\mathrm{C}, 5 \% \mathrm{CO}_{2}$ ). At the endpoint of incubation, supernatants were collected, filtered through $0.45 \mu \mathrm{m}$ filters and tested for the presence of IL-6, IL-12 and IFN- $\gamma$. Levels of individual cytokines were measured using a Quantikine mouse IL-6, IL-12 or IFN- $\gamma$ kit (R\&D Systems, Minneapolis, $\mathrm{MN})$.

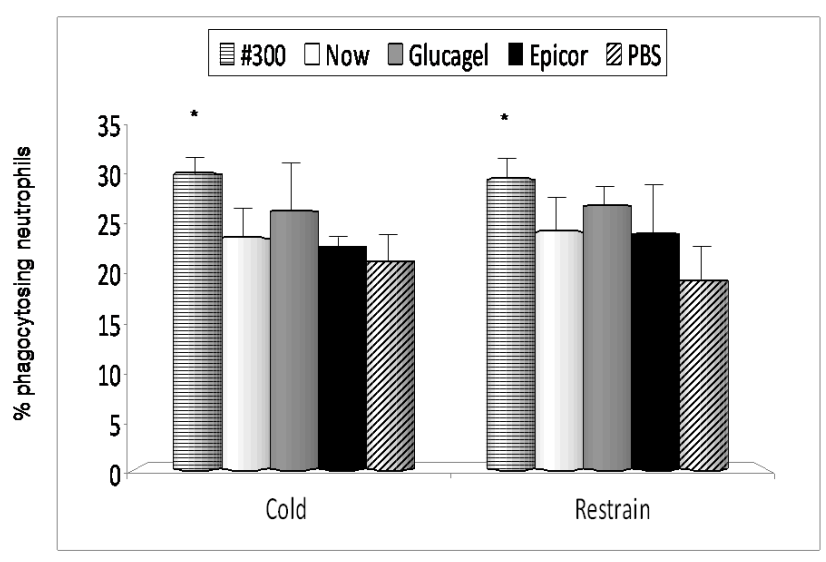

Fig. 1. Effect of an ip. administration of different glucan samples on phagocytosis by peripheral blood granulocytes and monocytes in stressed mice. Control level of phagocytosis (PBS only) in mice without stress was $34.3+2.2$.

* Represents significant differences between stressed-control (PBS) and glucan samples at $\mathrm{P} \leq 0.05$ level.

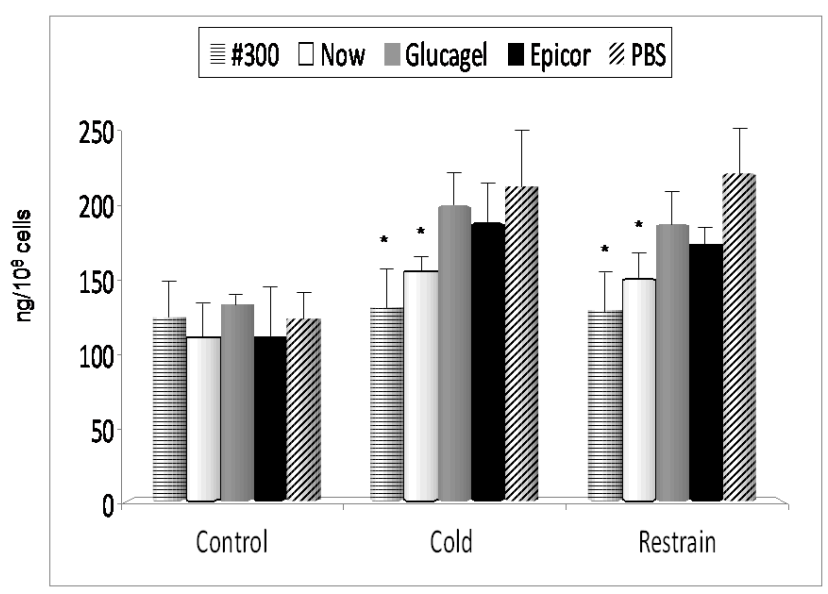

Fig. 2. Effect of 14 days of glucan feeding on stress-induced levels of corticosterone.

* Represents significant differences between stressed-control (PBS) and glucan samples at $\mathrm{P} \leq 0.05$ level.

\section{RESULTS}

The number of individual glucans is almost as great as the number of sources used for their isolation. The rationale for this combination of glucan samples was not only their commercial availability and success, but most importantly, we tried to include both soluble and insoluble glucans as well as glucans from different sources, including yeast, mushrooms and cereals.

Glucagel $\mathrm{T}$ barley $\beta$-glucan is a mixed link $(1 \rightarrow 3$, $1 \rightarrow 4)$ - $\beta$-D-glucose polymer, in which cellotriosyl and cellotetraosyl residues occur in a ratio of $\sim 3: 1$. The natural purification process yields a reduced molecular weight $\beta$-glucan (typically $\sim 130 \mathrm{kDa}$ ) that is more readily hydrat- 


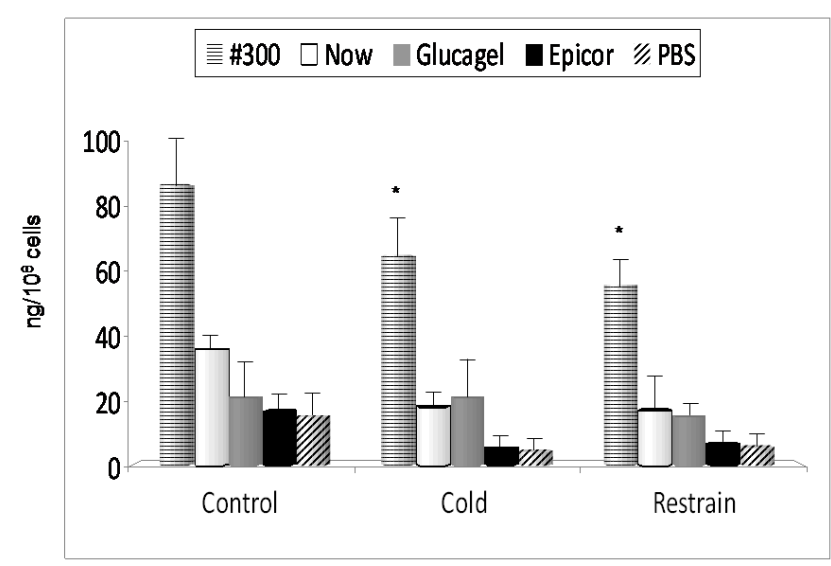

Fig. 3. Effect of 14 days of glucan feeding on stress-induced levels of IL-6.

* Represents significant differences between stressed-control (PBS) and glucan samples at $\mathrm{P} \leq 0.05$ level.

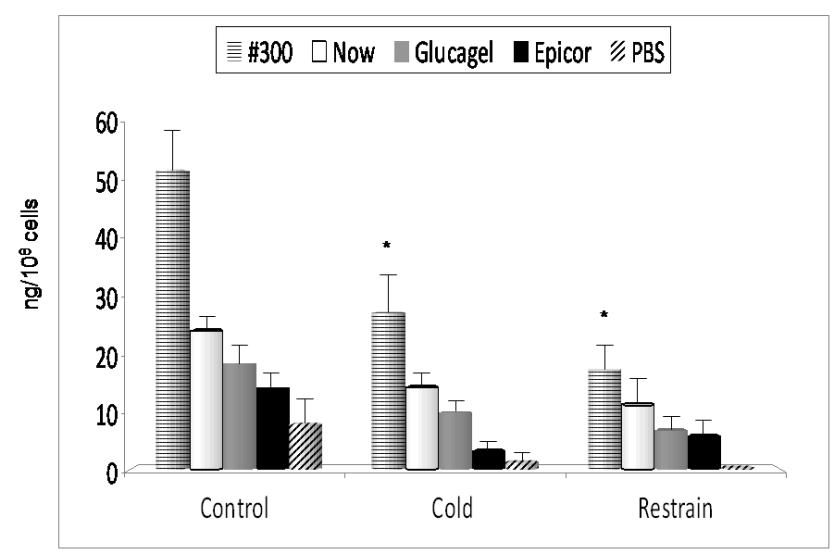

Fig. 4. Effect of 14 days of glucan feeding on stress-induced levels of IL-12.

* Represents significant differences between stressed-control (PBS) and glucan samples at $\mathrm{P} \leq 0.05$ level.

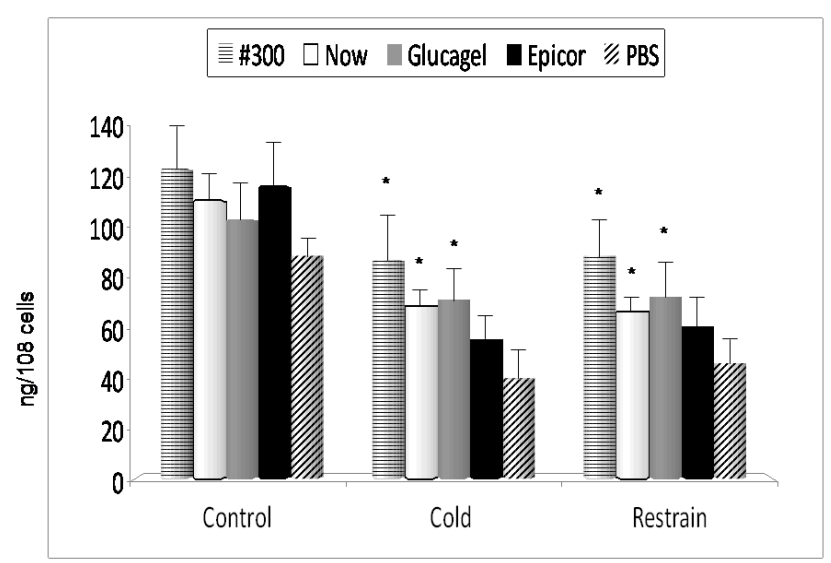

Fig. 5. Effect of 14 days of glucan feeding on stress-induced levels of IFN $\gamma$.

* Represents significant differences between stressed-control (PBS) and glucan samples at $\mathrm{P} \leq 0.05$ level. ed than other conventionally purified $\beta$-glucans. Glucan \#300 is a proprietary $(1 \rightarrow 3,1 \rightarrow 6)-B$-D-glucan purified from Saccharomyces cerevisiae by Biothera for Transfer Point and even when corresponding to the glucan sold under WGP name(s), the glucan \#300 has much higher purity (app. over 96\%). Epicor is also isolated from yeasts, however the information about its purity is not available. Now glucan is a mixture of both insoluble glucans from yeast and soluble glucans from mushrooms.

$\beta$-Glucans are generally considered to be potent stimulators of cellular immunity, with macrophages and neutrophils being the most important targets. Not surprisingly, the first experiments were focused on the role of glucan in stress-related changes in phagocytic activity. We used the synthetic polymeric microspheres (HEMA), since their use, dose and timing are already well established in glucan studies ${ }^{16}$. The results summarized in Fig. 1 show that cold stress caused a significant (38\%) decrease in phagocytic activity. While all glucans showed some ability to inhibit stress-related inhibition, only glucan \#300 was able to return the phagocytosis to a normal level. The same data were found in the case of restrainrelated stress (Fig. 1); however, the original inhibition of phagocytosis was stronger.

We then measured the level of corticosterone in serum. In the control group, feeding with glucans did not alter the level of corticosterone. On the other hand, both types of stress resulted in a significant increase of corticosterone, which was blocked to some extent by feeding with glucan. Glucans \#300 and Now were able to completely block the stress-related increase in corticosterone release (Fig. 2).

The basal production of IL- 6 by Con A-stimulated splenocytes was low and was significantly increased only by glucans \#300 and Now. Both types of stress reduced the IL-6 secretion, but only glucan \#300 managed to keep IL-6 secretion above control (PBS) levels (Fig. 3). The same results were obtained in the case of IL-12 (Fig. 4). In the case of IFN- $\gamma$, the glucans had almost no role in stimulating the secretion in control mice, but managed to boost the secretion to almost a normal level in stress-inhibited animals (Fig. 5). No differences were observed between food- and water-deprived control mice and normal control mice (data not shown).

\section{DISCUSSION}

Glucans belong to a group of physiologically active compounds, collectively termed biological response modifiers. In general, $\beta$-glucan is used as the chemical name of a polymer of $\beta$-glucose. In spite of being chemically heterogeneous, homopolymers of glucose having a linear molecule with (1-3)- $\beta$-D-glycosidic linkages or a branched one, with side chains bound by (1-6)- $\beta$-D-glycosidic linkages, are usually designated by the common name " $\beta$-glucans". There are various natural sources of $\beta$-glucans; however, they are most frequently prepared from yeast and fungal cell walls. 
Large numbers of individual glucans have been described in the literature. Due to the huge differences in activity among various glucans isolated from numerous sources, it is imperative to evaluate their biological properties before any suggestions for use of a particular glucan in clinical practice. This investigation focused on the role of four different types of commercially available glucan in restoring immune functions depressed by stress, as has been recently suggested ${ }^{14}$.

The effects of stress on the immune reaction are well established and are presumed to occur via the hypothalamic-pituitary-adrenal axis ${ }^{17}$ with corticosterone playing the major role ${ }^{18}$.

Not surprisingly, we started our evaluation of glucans by testing phagocytosis. We used the synthetic polymeric microspheres HEMA, since their use, dose and timing are already well established in glucan studies ${ }^{16}$. Compared to control values (over 34\% of peripheral blood neutrophils phagocytose these particles), both types of stress significantly decreased the phagocytic activity. All tested glucans increased the stress-induced suppression of phagocytosis, but only glucan \#300 caused significant differences.

In addition to the direct effect on various cells of the immune system, the immunostimulating action of $\beta$-glucans is caused by potentiation of a synthesis and release of several cytokines such as $\mathrm{TNF} \alpha$, IFN $\gamma$, IL-1 and IL-2. This cytokine stimulating activity is dependent on numerous factors including origin, molecular weight, and the triple helix conformation ${ }^{3}$.

The effects of glucans were tested on three different cytokines, secretion of which was inhibited by the stress. IL-12 is primarily secreted by cells on the macrophage lineage in response to microbial factors. IL-6 is pleiotropic cytokine produced by a variety of cells and IFN $\gamma$ is a cytokine critical for both innate and adaptive immunity. Regardless of the type cytokine tested, the situation was always similar - the production of cytokines was significantly inhibited by applied stress and feeding with glucan reversed this suppression. With exception of a yeast-derived glucan \#300 (and to some extent Now glucan), the reversion was not complete. It is important to note, however, that with the exception of IFN $\gamma$, each glucan stimulated cytokine secretion more than control mice fed with PBS. Kimura et al. ${ }^{14}$ found similar glucan-mediated restoration of stress-related inhibition of cytokine production. The differences in magnitude are most probably caused by differences among individual glucans.

Significant increase of corticostrerone levels after both types of stress are in agreement with previous studies ${ }^{14}$. Two types of glucan, \#300 and Now glucan, returned the corticosterone levels back to normal levels. This is hypothesed to help regulate the negative effects of stress.

Our current paper clearly demonstrates that glucans, particularly when sufficiently purified and well characterized, have pleiotropic effects reaching far beyond the originally suggested nonspecific modulation. Additional work is necessary to further evaluate the exact mechanisms of how glucans regulate the negative effects of stress. However, it is clear that glucan can be used orally, making it a significant dietary supplement with the potential for reducing or preventing stress.

\section{REFERENCE}

1. Benacerraf B, Sebestyen MM. Effect of bacterial endotoxins on the reticuloendothelial system. Fed Proc 1957; 16:860-7.

2. Rigi SJ, Di Luzio NR. Identification of a reticuloendothelial stimulating agent in zymosan. Am J Physiol 1961; 200:297-300.

3. Novak M, Vetvicka V. Beta-glucans, history and the present: Immunomodulatory aspects and mechanisms of action. J Immunotoxicol 2008; 5:47-57.

4. Novak M, Vetvicka V. Glucans as biological response modifiers. Endocrine Metabol. Immune Disorders-Drug Targets 2009; 9: 67-75.

5. Kojima T, Tabata K, Itoh W, Yanaki T. Molecular weight dependence of the antitumor activity of Schizophyllan. Agriculture Biol Chem 1986; 50:231-2.

6. Young S-H, Jacobs RR. Sodium hydroxide-induced conformational change in schizophyllan detected by the fluorescence dye, aniline blue. Carbohydrate Res 1998; 310:91-9.

7. Saitô H, Yoshioka Y, Uehara N, Aketagawa J, Tanaka S, Shibata Y. Relationship between conformation and biological response for (1-3)-beta-D-glucans in the activation of coagulation factor $\mathrm{G}$ from limulus amebocyte lysate and host-mediated antitumor. Carbohydrate Res 1991; 217:181-190.

8. Kabat EA. Structural Concepts in Immunology and Immunochemistry, Holt, Rinehart and Winston, New York, 1976.

9. Jamois F, Ferriéres V, Guégan J-P, Yvin J-C, Plusquellec D, Větvička V. Glucan-like synthetic oligosaccharides: iterative synthesis of linear oligo- $\beta$-(1,3)-glucans and immunostimularoty effects. Glycobiology 2005; 15:393-407.

10. Vetvicka V, Vetvickova J. An evaluation of the immunological activities of commercially available $\beta 1,3$ glucans. J Am Nutr Assoc 2007; 10:25-31.

11. Vetvicka V, Vetvickova J. A comparison of injected and orally administered glucans. J Am Nutr Assoc 2009; 11:42-8.

12. Vetvicka V, Fornusek L, Kopecek J, Kaminkova J, Kasparek L, Vranova M. Phagocytosis of human blood leukocytes: A simple micromethod. Immunol Lett 19982; 5:97-100.

13. Vetvicka V, Holub M, Kováru H, Siman P, Kováru F. Alphafetoprotein and phagocytosis in athymic nude mice. Immunol Lett 1987; 19:95-8.

14. Kimura Y, Sumiyoshi M, Suzuki T, Suzuki T, Sakanaka M. Effects of water-soluble low-molecular-weight $\beta$-1,3-D-glucan (branch $\beta-1,6)$ isolated from Aureobasidium pullulans 1A1 strain black yeast on restraint stress in mice. Pharmacy Pharmacol 2007; 59:1137-44.

15. Jainu M, Devi CSS. Antiulcerogenic and ulcer healing effects of Solanum nigrum (L.,) on experimental ulcer models: Possible mechanism for the inhibition of acid formation. J Ethnopharmacol 2006; 104:156-63.

16. Vetvicka V, Yvin J-C. Effects of marine $\beta$-glucan on immune reaction. Int Immunopharmacol 2004; 4:721-730.

17. Riley V. Psychoneuroendocrine influences on immune competence and neoplasia. Science 1981; 212:1100-9.

18. Dhabhar FS, Miller AH, Stein M, McEwn BS, Spencer RL. Diurnal and acute stress- induced changesin distribution of peritoneal blood leukocyte subpopulations. Brain Behav Immunol 1994; 8: 66-79. 